Indian J Anim Health (2021), 60(1) : 16-22

DOI: 10.36062/ijah.60.1.2021.16-22

\title{
African swine fever: An overview
}

\begin{abstract}
K. Sariga ${ }^{1 *}$ and P. M. Deepa ${ }^{1}$
${ }^{1}$ Department of Veterinary Epidemiology and Preventive Medicine, College of Veterinary and Animal Sciences, Pookode, Wayanad- 673 576, Kerala, India

Abstract

African swine fever (ASF) is a highly contagious viral disease of pigs, which causes about $100 \%$ mortality in domestic pigs. It creates huge economic loss through out the world although it has no zoonotic importance. For a long period, ASF remained endemic in Africa and few European countries but recently it has been reported in China and North Eastern states of India. The disease is transmitted to domestic pigs through contact with sick animals, infected wild boars consumption of contaminated feed and also by biological vectors (soft ticks of the genus Ornithodoros). The most common and suitable techniques to detect and identify ASFV is the polymerase chain reaction. The serological techniques play an important role in the surveillance program. Till date there is no effective vaccine developed so far, that is why most of the countries adopted the quarantine procedure and slaughter as effective control measures. This review throws light into the nature of the virus, epidemiology, transmission pattern, prevention and control of spread of the disease.
\end{abstract}

Key words: African swine fever, Outbreaks

\section{Introduction}

According to the release of the 2019 International Committee on Taxonomy of Viruses (EC 51, Berlin, Germany, July 2019), the family Asfarviridae has been included in the order of Asfuvirales and the class of Pokkesviricetes. Apart from this official nomenclature, it has been controversially discussed to include African swine fever virus (ASFV) into the tentative order of Megavirales containing the monophyletic but heterogeneous clade of nucleo-cytoplasmatic large DNA viruses (NCLDV).

The disease is characterized by disseminated hemorrhages on spleen, lymph nodes, kidney, gastrointestinal and respiratory tracts of swine causing a high mortality rate. ASFV affects both domestic and wild pigs of all breeds and ages. The wild suids and arthropod of the Ornithodoros genus are the natural hosts of this virus.

African swine fever virus replicates predominantly in the cytoplasm of infected cells (Iyer et al., 2006). The ASFV particles have a multilayered structure with an overall icosahedral morphology and a diameter of about $200 \mathrm{~nm}$. It is made up of a genomecontaining nucleoid, which is successively covered by a broad protein core-shell, an inner lipid membrane, an icosahedral protein capsid, and an outer lipid membrane. The virus enters into the cell by cell clathrin-mediated endocytosis and cell drinking mechanism (Salas and Andres, 2013). Neither proper treatment protocols nor vaccines are available for the prevention and control of the disease (Bastos et al., 2014).

An effective vaccine is not developed yet mainly due to the immuno-modulation developed by the virus which interferes with the cellular signaling pathway (Revilla et al., 2018). ASFV infection induces the modulation of Type-III interferon (IFN $\gamma$ ) production by $\mathrm{T}$ lymphocytes (Revilla et al., 1992). The most relevant source of IFN production in vivo after ASFV virulent infection is most likely dendritic cells (DCs) (Golding et al., 2016). ASFVencoded A238L, a multifunctional protein, has been shown to deregulate the level of proinflammatory molecules and cytokines by

\footnotetext{
*Corresponding Author
} 
inhibiting the activation of nuclear factor-kappa B (NF-kB) ((Neilan et al., 1997). CD2v another ASFV-encoded virus protein causes the adsorption of extracellular virus particles and red blood cells around virus-infected cells. $\mathrm{CD} 2 \mathrm{v}$ protein expression helps virus dissemination in pigs and it also has a role in impairing bystander lymphocyte function. This may be mediated either by direct interaction of $\mathrm{CD} 2 \mathrm{v}$ extracellular domain with ligands on lymphocytes or by an indirect mechanism involving interaction of the CD2v cytoplasmic tail with host proteins involved in signalling or trafficking pathways (Dixon et al., 2004). The unavailability of an applicable ASF vaccine is partly due to the complex nature of the virus, which encodes various proteins associated with immune evasion (Teklue et al., 2020).

\section{History}

ASF was first identified in Kenya in 1910 (Montgomery, 1921). After that disease has circulated in several African countries. In 1957 ASFV was introduced into Portugal through contaminated food waste from African airline flights and ships, which was fed to pigs and they controlled the outbreak by early detection of disease in the field followed by a rapid laboratory diagnosis, as well as enforcement of strict sanitary measures (Sánchez-Vizcaino et al., 2012). But it reestablished in 1960 in Portugal probably because of improper management of pig carcass which died of ASFV and spread to Spain (Bech-Nielsen et al., 1995). Portuguese veterinary services conducted a national campaign to the farmers by providing information of vector which was involved in the transmission of ASFV, detection of anti-tick antibodies in domestic and wild boars, as well as the destruction or isolation of the pig pens where ticks were present (Danzetta et al., 2020). Before it was finally eradicated in 1995, ASF remained endemic in the Iberian Peninsula (Sánchez-Vizcaino et al., 2012).

In 1978 ASF was spread and remained endemic in several parts of Sardinia (Mur et al.,
2016). Then the disease was reported from Europe and different countries of South and Central America. However, by utilizing rigorous disease control programs (biosecurity measures, awareness of disease, quarantine) it was successfully eradicated (Cwynar et al., 2019). For many years, ASF remained endemic only in African states and Sardinia (Costard et al, 2009). Though, in 2007 ASF was detected in Europe, namely Georgia, from where it was spread to neighbouring states Armenia, Azerbaijan and the Russian Federation (Sánchez-Vizcaino et al., 2013). During 20072017, more than 1,000 outbreaks have been reported and resulted in deaths of approximately 800,000 pigs in 46 regions across Russia (Sánchez-Vizcaino et al., 2013). There was a huge loss in pork production from 1,119 tons in 2007 to 608 tons in 2017 (Kolbasov et al., 2018). In 2012 and 2013, Ukraine and Belarus additionally revealed an ASF episode (SánchezVizcaino et al., 2015). In 2014, ASF surfaced at the European Union flare-ups in Lithuania, Latvia, Estonia and Poland (Gavier-Widen et al., 2015; Wozniakowski et al., 2016).

In August 2018, ASF was reported for the first time, in Shenyang, a north eastern city of China (Wang et al., 2018). As China is the world's largest pig producer, the outbreaks of ASF had a huge impact on both local and the world pig industry. The disease spread swiftly, reaching almost all the territories of the country. The disease might have been introduced from an ASF-affected Eastern European country via smuggled pork or offals (Wang et al., 2018). ASF has also spread beyond China within East and Southeast Asia (Normile, 2019). According to FAO's Emergency Prevention System for Animal Health, the disease had been reported in Vietnam, Cambodia, Mongolia, where millions of pigs were culled (FAO, 2019). At the end of 2018, Mongolia counted 27,819 domestic pigs (Heilmann et al., 2020). In Mongolia, the disease was reported in January 2019, with 11 outbreaks in 6 provinces involving 105 farms/ households. More than 10 per cent of the total pig population $(3,115)$ 
in Mongolia died due to the ASF outbreaks (FAO, 2019).

The Ministry of Agriculture and Rural Development (MARD) confirmed ASF outbreaks in Vietnam during February 2019, a total of 26 provinces reported outbreaks and more than 89,600 pigs were culled (FAO, 2019). Twenty-four genotypes of ASFV have been described to date, and all of them have been described in Africa. The causative strain belonged to p72 genotype II and was similar to the viruses identified in China and Georgia (Le et al., 2019). In April 2019 the Ministry of Agriculture, Forestry and Fisheries confirmed the first ASF outbreak in Cambodia, where more than 2400 pigs died or had been culled (FAO, 2019). The disease was also spread to the Philippines, Myanmar and Indonesia (OIE, 2020). ASF was first reported in South Korea in September 2019, and the virus strain (Korea/ Pig/Paju1/2019) belonged to the p72 genotype II with an intergenic region II variant, similar to those strains identified in Europe and China (Kim et al., 2020). In South Korea, the disease was spread in Eurasian wild boar populations like in Europe (Jo and Gortázar, 2020).

\section{Outbreak in India}

African swine fever has been first reported in the country in May 2020. There were 11 (7 from Assam and the remaining from the Arunachal Pradesh) outbreaks in North Eastern states bordering China (https://rr-asia.oie.int/en/ projects/asf/situational-updates-of-asf/) (OIE). Mortality of the pig was noticed in both Assam and Arunachal Pradesh reaching 100\% of the affected population and 1,199 pigs were found positive with ASFV out of 10,920 susceptible pigs. OIE referral laboratory, ICAR-National Institute for High Security Animal Diseases (NIHSAD), Bhopal confirmed the ASF by both Real-time PCR and nucleotide sequencing, further confirming the reported ASFV variant to be of genotype II origin (Abedin et al., 2020). In order to control the disease, the interaction between wild and domestic pig has been prevented. To keep the wild boars population safe from ASF infection, a trench of $2 \mathrm{~km}$ long and 6 feet deep was made near the World Heritage Site of Kaziranga National Park. A supervised zone circling the infected areas was created to restrict the movement of the pigs. Pig population in a $1 \mathrm{~km}$ radius around the infected area was tested and gradually culling was done. Transportation of pigs from other states like Meghalaya and Arunachal Pradesh was banned. Sanitization and awareness programs were conducted in different hot spots which were identified as core region for ASF (Bora et al., 2020). According to the 20th Livestock Census, there are 9.06 million pigs in the country, which is $1.7 \%$ of the total livestock in the country. North Eastern region has $46.85 \%$ of the pig population in the country. According to the OIE report on May 21, 2020, a total of 3701 pigs died due to ASF.

\section{Transmission}

ASF could be transmitted through direct contact with affected animals; indirect contact is also possible through contaminated food waste, feed garbage or fomites (Niederwerder et al., 2019) and also by biological vectors (soft ticks of the genus Ornithodoros). Experimentally the disease was transmitted through the intramuscular and oronasal route (Blome et al., 2013).

The natural sylvatic cycle, domestic pigtick cycle and domestic cycle are the three different epidemiological cycles that were described for ASFV transmission (Penrith et al. 2019). The wild boar-habitat cycle, which involves wart hogs (Phacochoerus africanus), was recently described in Europe (Chenais et al., 2018). Transmission of virus between the common warthog and argasid ticks (Ornithodoros moubata complex) is described under the natural sylvatic cycle (Chenais et al., 2018). The tick feeding on the blood of the warthog transmits the virus. It has been well documented in Southern and Eastern Africa. In the domestic cycle, transmission can occur through direct contact between infected and susceptible pigs, consumption of contaminated 
meat products and contact with fomites as well as when infected Ornithodoros ticks fed on them (Penrith et al., 2019). Warthog involvement is not there in the domestic cycle (Guinat et al., 2016).

\section{Clinical signs}

Domestic pigs and European wild boars showed a wide range of clinical signs from acute to chronic, where African wild pigs did not show any specific clinical signs (SánchezVizcaíno et al., 2019). In natural infection, incubation period ranges from 4 to 19 , depending on the virus dose and inoculation route (Mebus et al., 1981). Depending on the virulence of the isolate, exposure dose and route of infection, clinical manifestations vary. Highly virulent ASFV isolates may induce peracute and acute forms of the disease where moderately virulent isolates can produce acute, subacute and chronic or inapparent forms of the disease. Low virulent isolates may generate subacute or chronic or inapparent disease (Sánchez-Vizcaíno et al., 2019)

Peracute form: The peracute form of ASF is characterized by inappetence, body temperature $>41^{\circ} \mathrm{C}$, depression, and cutaneous hyperemia. Death usually occurs within 1-4 days after appearing clinical signs and symptoms (Sánchez-Vizcaíno et al., 2019).

Acute form: Affected animal shows high fever, fatigue and anorexia along with non-specific respiratory tract infection. Haemorrhages are observed on the skin around the ears, abdomen and legs. Diarrhoea along with other gastrointestinal symptoms are seen and death occurs within 6-13 days (or up to 20 days). Mortality rates may be as high as $100 \%$. Pregnant animals may abort (Sandra et al., 2020). In the final stages, rapid, labored breathing may be observed, as well as serous or seromucous nasal secretions caused by pulmonary edema (Sandra et al., 2020).

Apart from Sardinia, causative virus strains in Europe and Asia show high virulence for both domestic pigs and European wild boar under experimental conditions (Nurmoja et al.,
2017). In Sardinia, surviving animals were described with anti ASFV antibodies, intermittent viremia or subacute disease, as well as survivors did not show any clinical signs (Mur et al., 2016).

Chronic forms: This clinical form is characterized by multi-focal necrosis in the skin and arthritis, growth retardation, emaciation, respiratory distress and abortion, these signs are mainly associated with secondary bacterial infections inducing fibrinous polyserositis, chronic pneumonia, necrosis of skin, tongue or tonsils (Sánchez-Vizcaíno et al., 2015).

\section{Lesions}

Depending on the virulence factor of the virus, variation in the lesions may be observed in animals infected with ASFV. The important gross lesions are found in the kidney, spleen, lymph node and heart (Botija, 1982). The spleen may be enlarged, infarcted, friable and also darkened. The lymph nodes are hemorrhagic and it appears as red dark hematoma. Kidneys usually have petechial hemorrhages on cortical surfaces and cut surfaces as well as in the renal pelvis (Sandra et al., 2020).

Sub-acute form - it is a mild form of acute form. Hemorrhages are noticed in lymph nodes, kidneys and spleen, congested and edematous lungs. Sometimes interstitial pneumonia is also noticed (Mebus et al., 1981)

Chronic form - Fibrinous pericarditis, pleuritis, enlarged lymph nodes and spleen are the lesions found in chronic form. Caseous focal necrosis and mineralization are found in some cases (Mebus et al., 1981)

\section{Treatment options}

Till now no commercial vaccines are available, several antiviral agents are there. It is useful for early control of virus spread after an outbreak. Antivirals that are used against ASFV include resveratrol and oxyresveratrol, these antivirals achieved a $98-100 \%$ reduction in viral titers. Resveratrol at a noncytotoxic concentration of $5 \mu \mathrm{g} / \mathrm{mL}$ decreases viral 
production by $100 \%$. Oxyresveratrol at concentrations of $30 \mu \mathrm{g} / \mathrm{mL}$ decreases the viral production $98.5 \%$ for synthetic and $99.15 \%$ for natural oxyresveratrol. Both compounds permit early protein synthesis but prevented viral DNA replication, late viral protein synthesis and viral factory formation (Galindo et al., 2011).

Fluoroquinolones are an antibacterial agent but having antiviral activity. When ASFV-infected vero cells exposed to six different fluoroquinolones or their combinations, there was a severe reduction of the cytopathic effect from an early phase of infection (Mottola et al., 2013).

Cholesterol-lowering drugs or inhibitors of cholesterol are also antiviral agents. Accumulation of cholesterol in endosomes impairs fusion, resulting in the retention of virions inside endosomes (Cuesta et al., 2015)

\section{Control}

Many works have been done for developing a vaccine against ASF, attempts like vaccinate animals using extracts of infected cells, supernatants of infected pig peripheral blood leukocytes, inactivated and purified virions, infected glutaraldehyde-fixed macrophages, or detergent-treated infected alveolar macrophages have failed to induce protective immunity (Mebus, 1988). Currently, there are several reports of the protection elicited by experimental vaccines based in live attenuated ASFV (LAV) containing single or double gene deletions in the genome (O'Donnell et al., 2017), their different approaches including DNA vaccines (Reis et al., 2016). Some of them might turn into successful vaccines in future.

Countries which are free of disease followed prevention method like safe import policies (make sure to avoid illegal imports of live pigs and pork products, appropriate discarding of waste food from aircraft, vehicles or ships coming from infected countries ) and biosecurity.

Control measures during an outbreak will be difficult and must be adapted to the specific epidemiological situation. Sanitary measures may be employed including early detection and humane killing of animals (with proper disposal of carcass and waste); thorough cleaning and disinfection; zoning/ compartmentalisation and movement controls, surveillance and detailed epidemiological investigation; strict biosecurity measures on farms (OIE, 2020). In some places like Europe and Asia, a large number of wild boar population and unhygienic pig production are the main reason for the transmission of the disease. A good understanding and management of the wild boar population and good strategy among the veterinary services, wildlife and forestry authorities are required to successfully prevent and control ASF. The involvement of the soft tick vector, depending on the epidemiological situation should also be considered in the control program (OIE).

\section{Conclusion}

African swine fever disease has a consequential threat to the income and food security of huge numbers of people depending on the production and processing of pigs (FAO 2019). The disease has a high possibility for spreading into new areas where it can quickly develop and endure if initial detection and stern control measures are not applied. The disease distribution and recent spread to the Asian countries including India illustrate well the threat ASF poses to the pig production sector worldwide. ASF is having complicated epidemiology with the presence of different patterns, which depend on host and vector species densities and interrelationships, and are thus influenced by livestock production system characteristics and ticks and wild pig habitat. For that reason, prevention and control of the infection is needed along with a better understanding of its epidemiology, so that measures can be initiated.

Conflict of interest: Authors have no conflict of interest in this study. 


\section{REFERENCES}

Abedin SN, Baruah A, Bora A, Dutta D and Dutta A, 2020. African swine fever (ASF) outbreak in India: A review of literature about the virus and its control measures. Pharma Innovation, 9(7): 298-304

Bastos ADS, Fasina FO and King DP, 2014. African swine fever. Manual of security sensitive microbes and toxins. Taylor and Francis CRC Press: Boca Raton, FL, USA, pp 579-587

Bech-Nielsen S, Fernandez J, Martinez-Pereda F, Espinosa J, Bonilla QP et al., 1995. A case study of an outbreak of African swine fever in Spain. British Vet J, 151(2): 203-214, doi: 10.1016/S00071935(95)80012-3

Blome S, Gabriel C and Beer M, 2013. Pathogenesis of African swine fever in domestic pigs and European wild boar. Virus Res, 173(1): 122-130, doi: 10.1016/j.virusres.2012.10.026

Bora M, Bora D, Manu M, Barman NN, Dutta L et al., 2020. Assessment of risk factors of African swine fever in India: perspectives on future outbreaks and control strategies. Pathogens, 9(12): 1044, doi: 10.3390/pathogens9121044

Botija CS, 1982. African swine fever. New developments. Rev Sci Tech Off Int Epiz, 1(4): 1065 1094

Chenais E, Ståhl K, Guberti V and Depner K, 2018. Identification of wild boar - habitat epidemiological cycle in African swine fever epizootic. Emerg Infect Dis, 24(4), 810-812, doi: 10.3201/eid2404.172127

Costard S, Wieland B, de Glanville W, Jori F, Rowlands R et al., 2009. African swine fever: how can global spread be prevented? Philos Trans R Soc Lond B Biol Sci, 364(1530): 2683-2696, doi: 10.1098/ rstb.2009.0098

Cuesta-Geij MA, Chiappi M, Galindo I, Barrado-Gil L, Munoz-Moreno R et al., 2015. Cholesterol flux is required for endosomal progression of African swine fever virions during the initial establishment of infection. J Virol, 90(3): 1534-1543, doi: 10.1128/ JVI.02694-15

Cwynar P, Stojkov J and Wlazlak K, 2019. African swine fever status in Europe. Viruses, 11(4): 310, doi: $10.3390 / \mathrm{v} 11040310$

Danzetta ML, Marenzoni ML, Iannetti S, Tizzani P, Calistri P et al., 2020. African swine fever: lessons to learn from past eradication experiences. A systematic review. Front Vet Sci, 7: 296, doi: 10.3389/fvets.2020.00296

Dixon LK, Abrams CC, Bowick G, Goatle LC, KayJackson PC et al., 2004. African swine fever virus proteins involved in evading host defence systems. Vet Immunol Immunopathol, 100(3-4): 117-134, doi: 10.1016/j.vetimm.2004.04.002
FAO, 2019. GIEWS Update. East and Southeast Asia African swine fever is rapidly spreading in East and Southeast Asia threatening food security and livelihoods of households relying on pig farming, 2 July 2019. Rome, Italy: Food and Agriculture Organization of the United Nations

Galindo I , Hernáez B, Berná J, Fenoll J, Cenis JL et al., 2011. Comparative inhibitory activity of the stilbenes resveratrol and oxyresveratrol on African swine fever virus replication. Antiviral Res, 91(1): 57-63, doi: 10.1016/j.antiviral.2011.04.013

Gavier-Widén D, Gortázar C, Ståhl K, Neimanis AS, Rossi S et al., 2015. African swine fever in wild boar in Europe: A notable challenge. Vet Rec, 176(8): 199-200, doi: 10.1136/vr.h699

Golding JP, Goatley L, Goodbourn S, Dixon LK, Taylor $\mathrm{G}$ et al., 2016. Sensitivity of African swine fever virus to type I interferon is linked to genes within multigene families 360 and 505. Virology, 493: 15461, doi: 10.1016/j.virol.2016.03.019

Guinat C, Gogin A, Blome S, Keil G, Pollin R et al., 2016. Transmission routes of African swine fever virus to domestic pigs: current knowledge and future research directions. Vet Rec, 178(11): 262-267, doi: 10.1136/vr.103593

Heilmann M, Lkhagvasuren A, Adyasuren T, Khishgee B, Bold B et al., 2020. African swine fever in Mongolia: Course of the epidemic and applied control measures. Vet Sci, 7(1): 24, doi: 10.3390/ vetsci7010024

Iyer LM, Balaji S, Koonin EV and Aravind L, 2006. Evolutionary genomics of nucleo-cytoplasmic large DNA viruses. Virus Res, 117(1): 156-184, doi: 10.1016/j.virusres.2006.01.009

Jo YS and Gortázar C, 2020. African swine fever in wild boar, South Korea, 2019. Transbound Emerg Dis, 67(5): 1776-1780, doi: 10.1111/tbed.13532

Kim HJ, Cho KH, Lee SK, Kim DY, Nah JJ et al., 2020. Outbreak of African swine fever in South Korea, 2019. Transbound Emerg Dis, 67(2): 473-475, doi: 10.1111/tbed.13483

Kolbasov D, Titov I, Tsybanov S, Gogin A and Malogolovkin A, 2018. African swine fever virus, Siberia, Russia, 2017. Emerg Infec Dis, 24(4): 796798, doi: 10.3201/eid2404.171238

Le VP, Jeong DG, Yoon SW, Kwon HM, Trinh TBN et al., 2019. Outbreak of African swine fever, Vietnam, 2019. Emerg Infect Dis, 25(7): 1433-1435, doi: 10.3201/eid2507.190303

Mebus CA, 1988. African swine fever. Adv Virus Res, 35: 251-269, doi: 10.1016/S0065-3527(08)60714-9

Mebus CA, McVicar JW and Dardiri AH, 1981. Comparison of the pathology of high and low 
virulence African swine fever virus infections. In: Wilkinson PJ (edn.) African swine fever. EUR 8466 EN, Proceedings of the CEC/FAO research seminar, Sardinia, September 1981, pp 183-194

Montgomery RE, 1921. On a form of swine fever occurring in British East Africa (Kenya Colony). J Comp Pathol Therapeut, 34(3): 159-191, doi: 10.1016/S0368-1742(21)80031-4

Mottola C, Freitas FB, Simões M, Martins C, Leitão A et al., 2013. In vitro antiviral activity of fluoroquinolones against African swine fever virus. Vet Microbiol, 165(1-2): 86-94, doi: 10.1016/ j.vetmic.2013.01.018

Mur L, Atzeni M, Martínez-López B, Feliziani F, Rolesu S et al., 2016. Thirty five-year presence of African swine fever in Sardinia history evolution and risk factors for disease maintenance. Transbound Emerg Dis, 63(2): 165-177, doi: 10.1111/tbed.12264

Neilan JG, Lu Z, Kutish GF, Zsak L, Lewis TL et al.,1997. A conserved African swine fever virus IkappaB homolog, 5EL, is nonessential for growth in vitro and virulence in domestic swine. Virology, 235(2): 377-85, doi: 10.1006/viro.1997.8693

Niederwerder MC, Stoian AMM, Rowland RR, Dritz SS, Petrovan V et al., 2019. Infectious dose of African swine fever virus when consumed naturally in liquid or feed. Emerg Infect Dis, 25(5): 891-897, doi: 10.3201/eid2505.181495

Normile D, 2019. African swine fever marches across much of Asia. Science, 364(6441): 617-618, doi: 10.1126/science.364.6441.617

Nurmoja I, Petrov A, Breidenstein C, Zani L, Forth JH et al., 2017. Biological characterization of African swine fever virus genotype II strains from north eastern Estonia in European wild boar. Transbound Emerg Dis, 64(6): 2034-2041, doi: 10.1111/tbed.12614

O'Donnell V, Risatti GR, Holinka LG, Krug PW, Carlson J et al., 2017. Simultaneous deletion of the $9 \mathrm{gl}$ and $\mathrm{uk}$ genes from the African swine fever virus georgia isolate offers increased safety and protection against homologous challenge. J Virol, 91(1): e01760-16, doi: 10.1128/JVI.01760-16

Penrith ML, Bastos A, Charles Etter EM and BeltranAlcrudo D, 2019. Epidemiology of African swine fever in Africa today: Sylvatic cycle versus socioeconomic imperatives. Transbound Emerg Dis, 66(2): 672-686, doi: 10.1111/tbed.13117

Reis AL, Abrams CC, Goatley LC, Netherton C, Chapman DG et al., 2016. Deletion of African swine fever virus interferon inhibitors from the genome of a virulent isolate reduces virulence in domestic pigs and induces a protective response. Vaccine, 34(39): 4698-4705, doi: 10.1016/j.vaccine.2016.08.011

Revilla Y, Pérez- Núñez D and Richt JA, 2018. African swine fever virus biology and vaccine approaches. Adv Virus Res, 100: 41-74, doi: 10.1016/ bs.aivir.2017.10.002

Revilla Y, Pena L, Viñuela E, 1992. Interferon-gamma production by African swine fever virus-specific lymphocytes. Scand J Immunol, 35(2): 225-30, doi: 10.1111/j.1365-3083.1992.tb02854.x

Salas ML and Andrés G, 2013. African swine fever virus morphogenesis. Virus Res, 173(1): 29-41, doi: 10.1016/j.virusres.2012.09.016

Sánchez-Vizcaíno JM, Mur L and Martínez-López B, 2013. African swine fever (ASF): five years around Europe. Vet Microbiol, 165(1-2): 45-50, doi: 10.1016/j.vetmic.2012.11.030

Sánchez-Vizcaíno JM, Laddomada A and Arias ML, 2019. African swine fever virus. Diseases of swine, $11^{\text {th }}$ edn., pp 443-452, doi: 10.1002/ 9781119350927.ch25

Sánchez-Vizcaíno JM, Mur L and Martínez-López B, 2012. African swine fever: An epidemiological update. Transbound Emerg Dis, 59(Suppl 1): 27-35, doi: 10.1111/j.1865-1682.2011.01293.x

Sánchez-Vizcaíno JM, Mur L, Gomez-Villamandos JC and Carrasco L, 2015. An update on the epidemiology and pathology of African swine fever. J Comp Pathol, 152(1): 9-21, doi: 10.1016/ j.jcpa.2014.09.003

Sandra B, Kati F and Martin B, 2020. African swine fever - A review of current knowledge. Virus Res, 287(2): 198099, doi: 10.1016/j.virusres.2020. 198099

Teklue T, Sun Y, Abid M, Luo Y and Qiu HJ, 2020. Current status and evolving approaches to African swine fever vaccine development. Transbound Emerg dis, 67(2): 529-542, doi: 10.1111/tbed.13364

Wang T, Sun Y and Qiu HJ, 2018. African swine fever: An unprecedented disaster and challenge to China. Infect Dis Poverty, 7(1):111, doi: 10.1186/ s40249-018-0495-3

World Organisation for Animal Health (OIE), 2020. Available in https://www.oie.int/en/disease/africanswine-fever/ (Available on 24.04.2021)

Wozniakowski G, Kozak E, Kowalczyk A, Lyjak M, Pomorska-Mol M et al., 2016. Current status of African swine fever virus in a population of wild boar in eastern Poland (2014-2015). Arch Virol, 161: 189- 195, doi: 10.1007/s00705-015-2650-5

Received - 02.02.2021, Accepted - 19.04.2021, Published-01.06.2021

Section Editor: Prof. S. N. Joardar, Associate Editor 